\title{
(DIE) - MODELO PARA EL DISEÑO DE IDEAS DE EMPRENDIMIENTO
}

\author{
(DBI) Model for the design of business ideas
}

\section{Artículo de Investigación}

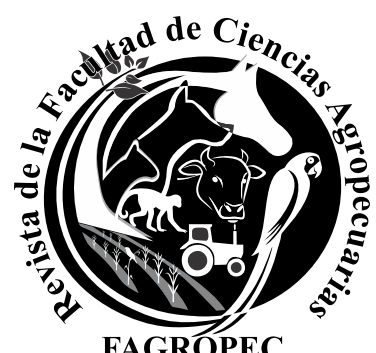

Recibido 15 de enero de 2020. Aceptado 3 de marzo de 2020.

${ }^{1}$ MSc., Universidad Francisco de Paula Santander, Grupo de Investigación GI@SD y GIPAB

${ }^{2}$ MSc., Universidad Francisco de Paula Santander, Grupo de Investigación GI@SD y GIPAB

${ }^{3}$ MSc., Universidad Francisco de Paula Santander, Grupo de Investigación GIPAB

${ }^{4}$ Universidad de Santander UDES, Facultad de Ciencias Económicas, Administrativas y Contables, Estudiante Administración Financiera

${ }^{5}$ Universidad de Santander UDES, Facultad de Ciencias de la Salud, Estudiante del programa de Bacteriología y laboratorio Clínico

${ }^{6}$ Universidad de Pamplona, Facultad de Ciencias Agrarias, Programa de Zootecnia,

\section{Como citar:}

VELÁSQUEZ CARRASCAL, Blanca Liliana. et al. (DIE) Modelo para el diseño de ideas de emprendimiento. En: Revista Facultad Ciencias Agropecuarias - FAGROPEC. Universidad de la Amazonia, Florencia Caquetá. Volumen 12 enero-junio, 2020. Pp. 52-64 ISSN en Línea: 2539-178X. https://doi.org/10.47847/fagropec.v12n1a5

*Autor para Correspondencia: blvelasquezc@ufpso.edu.co
Blanca Liliana Velásquez Carrascal ${ }^{1^{*}}$, Johann Fernando Hoyos Patiño $^{2}$, Daniel Antonio Hernández Villamizar ${ }^{3}$, Lilian Natalia Sayado Velasquez ${ }^{4}$, Jeimmy Estefany Sayago Velásquez ${ }^{5}$, José Antonio Vargas Yuncosa ${ }^{6}$,

\section{RESUMEN}

Los jóvenes en la actualidad deben responder a las exigencias del mercado actual creando iniciativas de negocios claras y ordenadas, con un proceso que genere prototipos de empresas innovadoras a través de un paso a paso que responda a la realidad del entorno, el objetivo de la investigación es describir el modelo para el Diseño de Ideas de Emprendimiento (DIE) que consta de 8 fases; concepto del negocio, propuesta de valor, análisis de mercado, plan de mercadeo, estructura administrativa, estudio técnico y operativo, planificación financiera y resumen ejecutivo. Es una herramienta metodológica para estudiantes que permite la construcción del plan de negocio o proyecto empresarial, aumentando el porcentaje de viabilidad al momento de la ejecución y puesta en marcha de la empresa.

\section{Palabras claves:}

Emprendimiento; Negocio; Empresa; Innovación

\begin{abstract}
In this days, young people must be able to respond to the demands of the current market developing clear and orderly business initiatives, in a process capable to generates prototypes of innovative companies through a step-bystep approach that responds to the reality of the environment, the objective of this research is to describe the model for the Design of Entrepreneurial Ideas (DIE) which consists of 8 phases; business concept, value proposal, market analysis, marketing plan, administrative structure, technical and operational study, financial planning and executive summary. It's a methodological tool for students that allows the construction of the business plan or business project, increasing the percentage of viability at the time of execution and implementation of the company.
\end{abstract}

Key words:

Entrepreneurship; Business; Enterprise; Innovation 


\section{INTRODUCCIÓN}

Las instituciones educativas en Colombia se esfuerzan cada día desde sus programas educativos para promover el desarrollo del espíritu emprendedor desarrollando estrategias pedagógicas apoyadas en la creatividad y la innovación, según Gómez \& Mitchell (2014). Manifiesta que la creatividad no se da de forma casual, esta debe ir acompañada de un proceso que estimule la generación de ideas propiciando ambientes para ello. Es por esta razón que el programa de Zootecnia del Francisco de Paula Santander Ocaña (UFPSO) desarrolla por medio de las asignaturas de la línea administrativa un modelo para la creación de ideas de negocios que apuntan al desarrollo económico del sector agropecuario.

Según Kirberg (2014) El emprendimiento es un término que ha estado presente en la historia de la humanidad por décadas buscando estimular las sociedades para la generación de empresas, en los últimos años se utiliza como estrategia para incrementar la búsqueda de nuevas oportunidades empresariales con propuestas de valor generando empleos en las regiones que favorezca el desarrollo económico de las mismas.

A través del emprendimiento se pretende motivar la creación de empresas que fortalezcan el desarrollo económico del país según Chamorro, et al., (2013). Es por esta razón que en Colombia el Congreso de la República (2006) a través de la ley 1014 del 2006 se busca el fomento de la cultura del emprendimiento y su decreto reglamentario 4463 del 15 de diciembre del 2006 con el propósito de motivar las instituciones educativas de todo orden y nivel de formación en la importancia del emprendimiento para los grandes retos de la globalización económica, Vásquez (2011). Razón por la que se deben promover la identificación de nuevas ideas de negocios apoyados en la creatividad, innovación y uso de tecnologías que fortalezcan el tejido empresarial en el país.

En Colombia se estableció de forma obligatoria a través de la ley 1014 del 2006 que las instituciones de educación promuevan la enseñanza del emprendimiento buscando que los niños y jóvenes desarrollen las competencias y las habilidades para la formación de futuros empresarios. De igual forma Hernández \& Rodríguez (2013) indican que la academia desde sus distintos niveles de formación mantiene en una búsqueda constante de estrategias pedagógicas para el desarrollo de nuevas ideas creativas empresariales. Por lo anterior expuesto el programa académico de zootecnia de la UFPSO en la formación del futuro profesional fortalece las habilidades y conocimientos que estimulan el espíritu emprendedor, diseñando mecanismo que inquieten al estudiante en propuestas para creación de empresa y entre ellas la opción de elaborar un plan de negocio como trabajo de grado.

Con esta estrategia el programa de zootecnia busca fortalecer durante el desarrollo de las competencias en gestión empresarial la madurez de una idea de negocio que sus estudiantes trabajen desde la línea administrativa asociada con la línea productiva, articulando estas asignaturas con la intensión de que sus estudiantes alcancen los objetivos trazados en su proyecto de vida incluyendo el emprendimiento en la construcción de su perfil profesional. De acuerdo con Rodríguez (2006, p. 15) la actitudes emprendedoras se estimulan por medio de la generación de ideas innovadoras y creativas que se puedan transformarse y fortalecer durante el tiempo trabajado. 
Preparar profesionales que se enfrenten a los retos que exige la economía actual donde la incertidumbre es una constante, es el desafío a que sume del programa de zootecnia UFPSO propiciando espacios para que sus estudiantes generen ideas de negocios que se sostengan en los mercados de forma exitosas. De acuerdo con Schnarch (2014) en los tiempos actuales se motiva el espíritu emprendedor a través de cursos, talleres y actividades académicas que con lleven a identificar ideas de emprendimiento exitoso.

Por otro lado Mackinlay \& Sabbagh, (2011) manifiestan que es importante implementar técnicas que ayuden a la búsqueda de ideas creativas que ayuden a minimizar la incertidumbre frente al desarrollo de un proyecto productivo, venciendo el temor que produce para el emprendedor enfrentarse a un reto empresarial. Campos (2007) indica que es una tendencia para las universidades formar profesionales generadores de empleos que puedan transformar la realidad del país, creando vínculos con el sector productivo de manera que sus estudiantes se adentren a una realidad en los negocios del mercado actual.

El modelo educativo del programa de zootecnia busca brindar una respuesta al entorno económico de la región formando profesionales con altas capacidades y habilidades en emprendimiento que asuman de forma creativa los cambios que manifiesta el mercado. Kirkberg (2013) menciona que la innovación y la creatividad en el economía se deben integran para la creación de empresas que puedan desarrollar y lanzar nuevos productos y servicios con factores de éxito, trabajados a través de metodologías que brinde apoyo para mitigar el riesgo a un fracaso.

La identificación de dicha metodología que desde la formación académica en las instituciones educativas pueda despertar el espíritu emprendedor vinculando las diferentes asignaturas de la malla curricular del programa es un desafío. Para Campo \& Albarracín (2008), citado por Forero (2014) la relación en el desarrollo de las actitudes del emprendimiento con las diferentes ciencias de forma directa como la economía y de forma indirecta como la sociología y la biología, permite reconocer que es posible diseñar un modelo creativo que fomente la creación de ideas de negocios. Es por esta razón que el programa de zootecnia UFPSO crea un modelo desde su maya curricular para el desarrollo y análisis de nuevas ideas de negocios.

\section{MATERIALES Y MÉTODOS}

El enfoque de la investigación cualitativa se caracteriza recopilar información del comportamiento cultural, comprender y reconocer la experiencia vivida, de acuerdo con Patiño \& Gómez (2016). Por lo anterior se buscó recopilar información del proceso para la generación de ideas de negocios de los estudiantes del programa de zootecnia UFPSO desde el desarrollo de las diferentes asignaturas de la línea administrativa de su malla curricular.

Según Hoyos, et al., (2019) La investigación de tipo descriptivo consiste en reseñar las características del fenómeno del grupo analizando. Reconociendo las principales características de los aspectos claves para el desarrollo de las propuestas de negocio de los estudiantes.

El método consiste en el desarrollo de 8 fases que facilite al estudiante identificar las características conceptuales y numéricas claves de éxito en la creación de su idea de negocio. De acuerdo con 
Madariaga (2019) se debe seguir una ruta clara y planificada que le brinde los parámetros al emprendedor para enfrentar el reto de desarrollar y modelar por etapas paso a paso su idea de negocio fundamentada en procesos de investigación y aprovechamiento del potencial de la región.

\section{RESULTADOS Y DISCUSIÓN}

El modelo para el diseño de ideas de emprendimiento (DIE) pretende mediante el desarrollo académico del estudiante generar y concretar planes de negocios fomentando las habilidades que permitan el reconocimiento de oportunidades en el mercado actual construyendo cultura empresarial en sus estudiantes.

Para Colomina \& Yáñez (2014) es importante que el emprendedor aplique una herramienta siguiendo un proceso para validar y definir las ideas de negocios desde su componente conceptual y numérico con una estructurara práctica y sencilla, Por esta razón crearon el método para validación y análisis de Ideas de negocio (AVIN). De acuerdo con Trimi \& Berbegal (2012) la construcción de una herramienta que facilite el diseño de un modelo de negocio le da la oportunidad al emprendedor de definir el producto o servicio de la empresa nueva estableciendo limites en criterios relevantes para el entorno donde se desarrollen.

El DIE nace como estrategia pedagógica que ayude en la orientación de los estudiantes en el modelado de ideas negocios con ventajas competitivas en la región. De acuerdo con Duarte \& Tibana (2009) quienes generan ideas innovadoras causan un impacto social y económico aumentando las posibilidades de crecimiento económico en el entorno. El programa de zootecnia UFPSO desarrolla el DIE como una herramienta que se apoya en las asignaturas de la línea administrativa de la maya curricular. Según Galvis (2007) las universidades públicas o privadas buscan espacios académicos que fomenten el emprendimiento observando que los resultados de la evolución de las ideas de negocios propician desde las prácticas pedagógicas de la docencia como actor fundamental y eje transversal de la malla curricular.

La UFPSO desde el programa de zootecnia cuenta con una línea administrativa que fortalece las capacidades y habilidades de los estudiantes de manera progresiva generan nuevas ideas empresariales innovadoras y creativas. Según Campos \& Gómez (2016) las universidades deben proporcionar herramientas a los alumnos en el desarrollo de su carrera para que a futuro como profesionales logren enfrentarse a un mundo globalizado con las exigencias de los mercados actuales en la creación de negocios competitivos. En la Figura 1 se representa la línea administrativa de la malla curricular del programa de Zootecnia UFPSO.

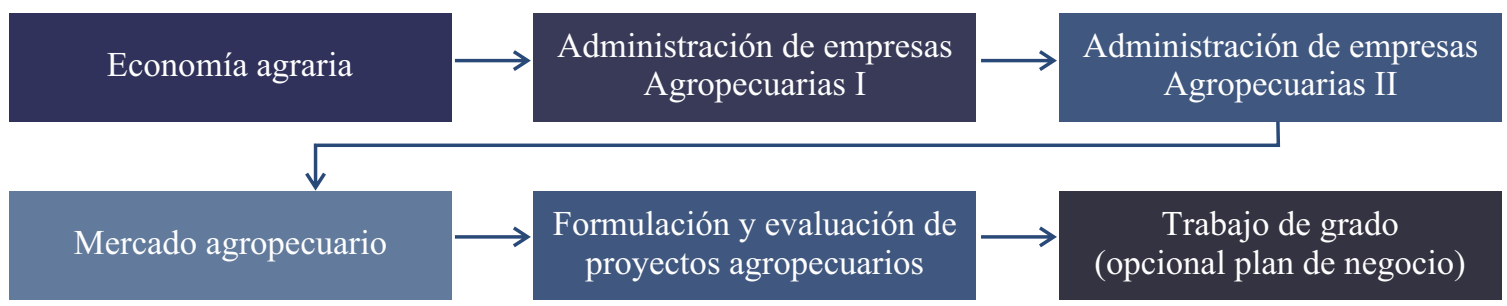

Figura 1. Línea administrativa programa de Zootecnia UFPSO 
El modelo DIE, apoyado en la malla curricular pretende que el estudiante desarrolle de forma estructurada y organizada la planeación de su idea de negocio en 8 etapas, siguiendo los lineamientos del ministerio de Comercio Industria y Turismo de Colombia (2010), ajustándolos a la realidad del entorno actual de los estudiantes del programa. En la Figura 2 se muestra de forma gráfica el (DIE) Modelo para el diseño de ideas de emprendimiento del programa de Zootecnia UFPSO.
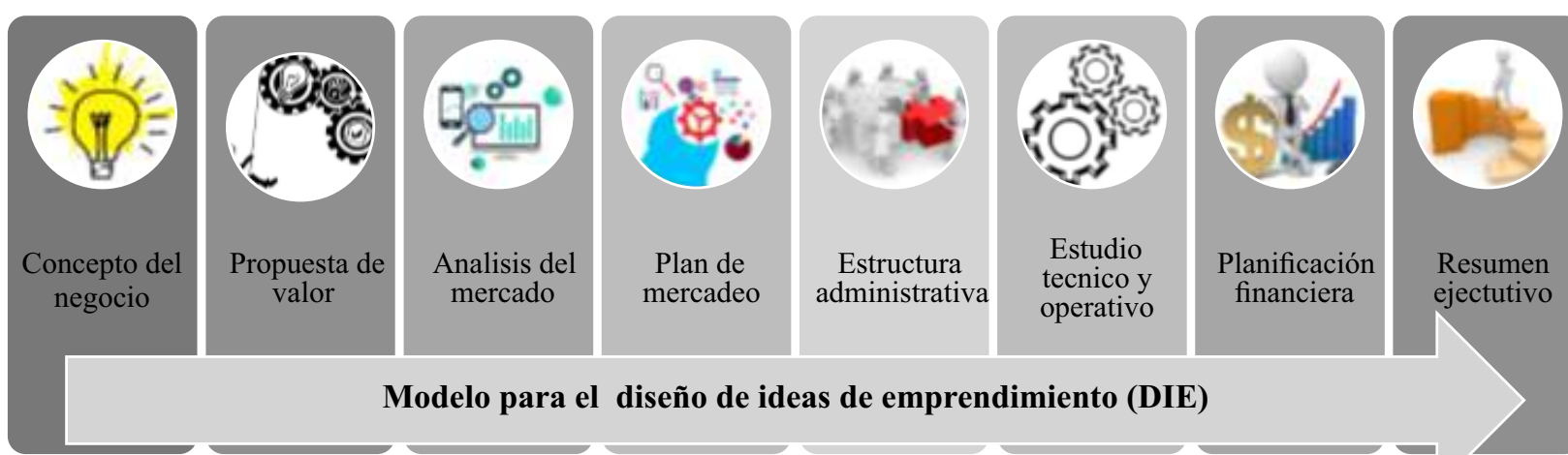

Modelo para el diseño de ideas de emprendimiento (DIE)

Figura 2. Modelo para el diseño de ideas de emprendimiento (DIE)

\section{Etapas del modelo para el diseño de ideas de emprendimiento (DIE)}

\section{Etapa 1 Concepto del negocio}

Los estudiantes una vez seleccionan la idea de emprendimiento, deben definir el producto o servicio, los objetivos de la empresa, las características de la misma, localización y ubicación. De acuerdo con Rendon (2014) en su modelo de plan de negocio para micro y pequeña empresa la primera fase debe contener la descripción del negocio además del portafolio de los productos o servicios que están dispuesto a ofrecer. En la Figura 3 se muestra la etapa uno del DIE que consiste en describir el concepto del negocio ilustrada de forma gráfica.

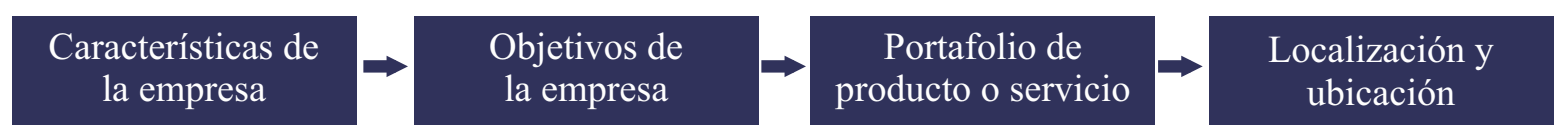

Figura 3. concepto del negocio etapa 1 del DIE

De acuerdo Pérez \& Darío (2014). El Servicio Nacional de Aprendizaje (SENA) forma a sus aprendices bajo el programa Jóvenes Rurales Emprendedores desarrollando un modelo pedagógico que conlleva a la creación de escenarios en búsqueda de negocios innovadores. A sí mismo la UFPSO pretende inquietar a sus estudiantes para que aporten a la competitividad económica apostándole a la caracterización sus ideas empresariales aprovechando las oportunidades regionales, departamentales, nacionales e internacionales.

\section{Etapa 2 propuesta de valor}

Según Villarán (2009), citado por Morcela et al., (2018) la propuesta de valor consiste en reconocer las características distintivas de los productos o servicios y su ventaja competitiva ante la 
competencia. En la figura 4 se muestra la ruta que el estudiante debe seguir para estructurar la propuesta de valor como segunda etapa del DIE.

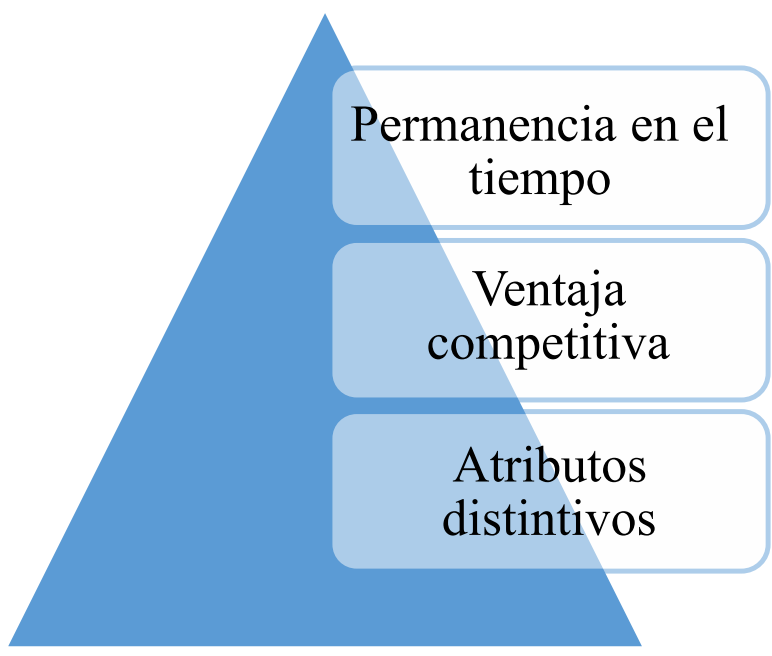

Figura 4. propuesta de valor etapa 2 del DIE

La propuesta de valor en el DIE pretende definir las características que hacen diferente los productos y servicios, reconocer cual sería la ventaja competitiva de la idea de negocio y las estrategias que se implementaran para mantenerlo de forma exitosa en el tiempo. De igual manera de acuerdo con Luna et al., (2018) es importante contemplar en los productos o servicios la elaboración sana $\mathrm{y}$ sostenible como propuesta de valor.

\section{Etapa 3 Análisis de mercado}

De cuerdo Balanko (2008) citado por Bravo (2015) un factor que segura el éxito de para la creación de las nuevas empresas es realizar una investigación cuidadosa de los aspectos relevantes del mercado. Es por esta razón que el DIE contempla en su tercera etapa el análisis de mercado que tiene como propósito reconocer los clientes potenciales, comportamiento del consumidor, análisis de la demanda, análisis de la oferta, análisis de la competencia y los canales de distribución. En la figura 5 se muestra la ruta que el estudiante debe seguir para estructurar el análisis de mercado como tercera etapa del DIE.

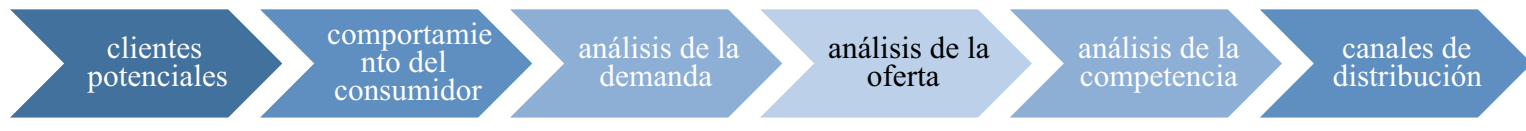

Figura 5. Análisis de mercado etapa 3 del DIE

El propósito del estudio de mercado es determinar la tendencia del mercado, identificando el comportamiento del consumidor, la demanda y oferta que permita elegir el precio y canal de distribución del producto o servicio para el nuevo modelo de negocio. Según Gómez et al., (2018). es fundamental para una empresa conocer el comportamiento del consumidor que brinde un panorama sobre las tendencias del mercado objetivo.

\section{Fase 4 plan de mercadeo}

Para Vargas (2002) citado por Barsky (2016) en la identificación de un modelo de negocio se debe dar gran relevancia a las estrategias que se plasmara en el plan de mercadeo. Así mismo el DIE en su cuarta etapa conduce a diseñar las estrategias que tienen como propósito aumentar el crecimiento del mercado meta del negocio. De acuerdo con Mora et al,. (2019) la producción de tácticas innovadoras que conlleven a los nuevos modelos de negocio posesionarse frente a las empresas competencia del mercado. En la figura 6 reconoce los aspectos a los que apuntan el plan de marketing de la cuarta etapa del DIE. 


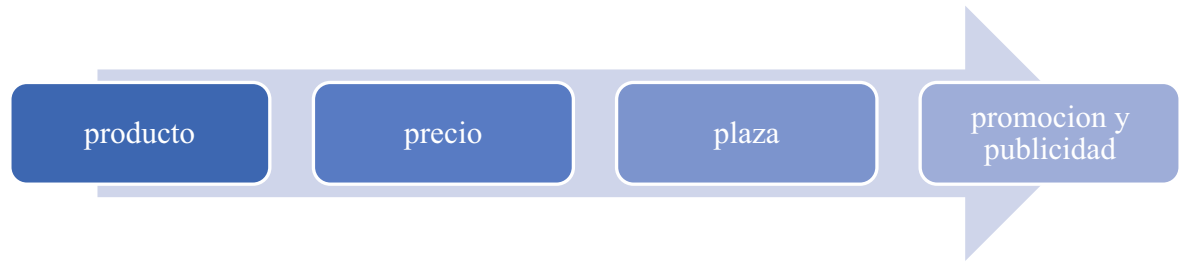

Figura 6. Análisis de mercado etapa 4 del DIE.

Según Fuentes et al,. (2019) en el desarrollo del plan de negocios para una aplicación entre amigos que puedan reunir dinero, contemplan un plan de marketing planeando estrategias desde los productos que se ofrecen, precios, mercado y la publicad del mismo. Por tal razón DIE contempla la planificación de las estrategias de mercadeo. De acuerdo con Chacón et al., (2018). Se deben buscar nuevas formas para distribuir productos en el mercado con el uso de nuevas tecnologías.

\section{Fase 5 estructura administrativa}

De acuerdo con Castro (2017) el grupo de trabajo requiere definir las características de acuerdo del modelo de negocio que proyecta. El DIE orienta al emprendedor para que reconozca el capital humano necesario en su modelo de negocio, identifique los requisitos, funciones y responsabilidades de cada cargo proyectado para la estructura organizacional. En la figura 7 plasma la estructura administrativa y organizacional de la nueva empresa como quinta etapa del DIE.

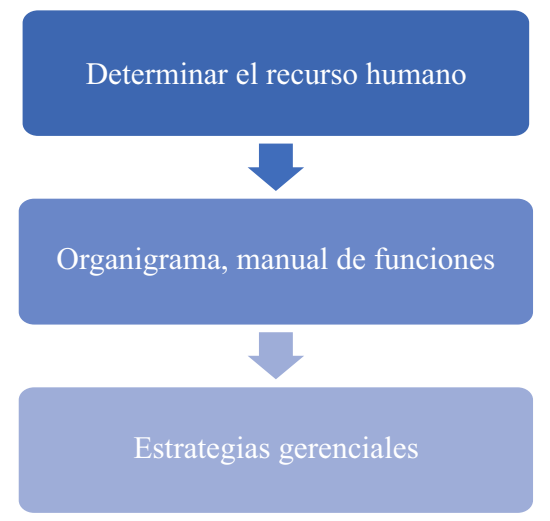

Figura 7. Estructura administrativa etapa 5 del DIE

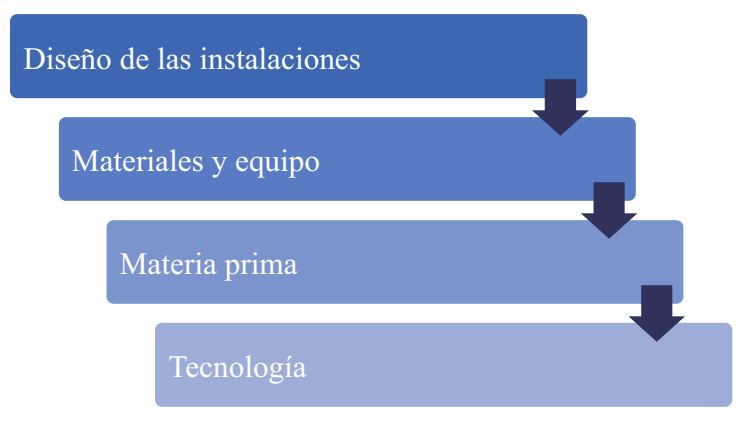

Figura 8. Estudio técnico y operativo etapa 6 del DIE.
De acuerdo con Cedeño et al,. (2019) en la administración de los negocios es clave implementar estrategias gerenciales que permita la ventaja competitiva en la economía. La estructura administrativa del DIE reconoce la importancia de la filosofía de gestión empresarial; misión, visión y valores instituciones, además de los niveles de autoridad, funciones y responsabilidades de cada cargo requerido en el funcionamiento del negocio así como el estilo de la dirección.

\section{Fase 6 Estudio técnico y operativo}

Uribe (2015), determinar la capacidad instalada que requiere el montaje de la empresa se encuentra dentro de la etapa de perfectibilidad que se propone como los pasos que se deben seguir para la proyección de la nueva empresa. En la figura 8 el DIE propone identificar las instalaciones, la maquinaria y equipo, materias primas y la tecnología requeridas para el funcionamiento de la empresa. En la figura 8 el DIE propone identificar las instalaciones, la maquinaria y equipo, materias primas y la tecnología requeridas para el funcionamiento de la empresa. 
De acuerdo a las fases del DIE el estudio técnico operativo define Básicamente en el estudio técnico se define aspectos que deben contener el plano de las instalaciones, los materiales maquinaria, equipo y tecnologías necesarias para el funcionamiento del proyecto.

Para Santandreu et al,. (2014) citado por López (2017) existen modelos para planes de negocios como el Canvas, que propone 9 pasos en un lienzo donde se estructuran los aspectos que desarrollan la propuesta de negocio, uno de estos aspectos pide al emprendedor plasmar los recursos físicos necesarios para el funcionamiento de su negocio. Razón por la cual el modelo DIE contempla como paso importante de la herramienta la identificación de los insumos, materias primas, maquinaria, equipo, tecnologías y diseño y distribución de las instalaciones donde funcionara la empresa.

\section{Fase 7 Planificación financiera}

Según Stutely (2000), citado por Ferreira (2015) en un negocio nuevo se recomienda identificar las fuentes de financiación del proyecto, los costos de producción y la inversión necesaria para su puesta en marcha. Para el DIE la estructura de los recursos financieros le concederá al emprendedor la oportunidad de proyectar la rentabilidad de su negocio. Por otro lado Ascanio et al., (2018) indican que una vez se proyecta la descripción financiera, se debe realizar una evaluación que muestre la rentabilidad de la empresa. En la figura 9 el DIE proyecta la planificación financiera, calculando Presupuesto de Inversión, ingresos, costos y gastos para posteriormente analizar la rentabilidad del negocio.
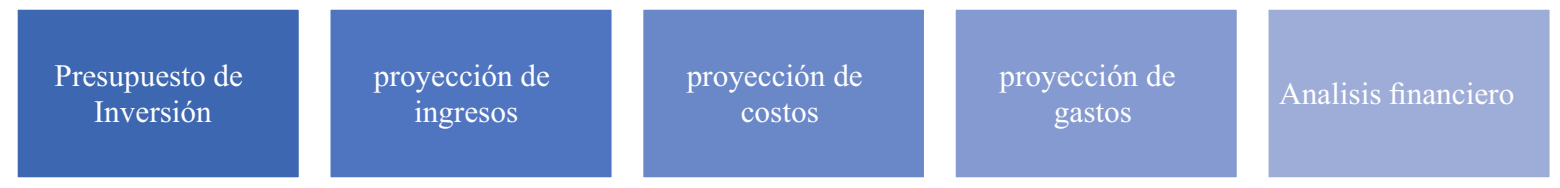

Figura 9. Planificación financiera etapa 7 del DIE.

De acuerdo con Vázquez \& Pinzón (2016) donde proponen como herramienta para la elaboración de un plan de negocio el (BSC) Cuadro de Mando Integral, una de sus cuatro etapas que dan forma a la propuesta es la proyección financiera. Es por esto que siguiendo la propuesta de los autores mencionados el DIE contempla dentro de su modelo el cálculo de las ventas de bienes o servicios para el reconocimiento del presupuesto de inversión, ingresos, cotos de producción y funcionamiento, gastos de operación y como último paso realizar un estudio para determinar la sostenibilidad y rentabilidad del negocio. Como lo manifiestan Rubio et al, (2019) la planeación financiera muestra escenarios económicos claros y reducen la incertidumbre de riesgo.

\section{Fase 8 Resumen ejecutivo}

Mercedes et al, (2017). El emprendedor debe ser creativo para expresar de forma asertiva y concisa su idea de negocio. El DIE condesa de forma corta en la fase ocho el resumen ejecutivo que muestre los componentes claves; concepto del negocio, análisis de mercado, plan de mercadeo, estructura organizacional, estudio técnico y operativo, análisis financiero) de la idea de negocio.

\section{CONCLUSIONES}

Implementar el modelo DIE en la formación del zootecnista optimiza las competencias en las áreas de desempeño y la incursión en nuevos espacios laborales, apoyados en la malla académica del 
programa estimulando habilidades y competencias en el enfoque administrativo y gerencial del futuro profesional.

Con la aplicación del modelo DIE se priorizara el estudio de mercado como punto fundamental de la cadena productiva, definiendo de esta forma las calidades y cantidades para suplir la demanda del entorno, además permitiendo a los estudiantes planear de forma organizada y estructurada sus ideas de negocio, adicionalmente crear estrategias innovadoras que les ayuden a mantener con éxito en mercados actuales reduciendo en gran parte las posibilidades de fracaso.

Las instituciones educativas tienen el compromiso de formar profesionales que respondan a las necesidades del contexto actual de la sociedad, es por esto que nace el modelo DIE, como estrategia pedagógica para fomentar el espíritu emprendedor de los estudiantes del programa de zootecnia UFPSO, aportando al desarrollo sostenible de la región, respondiendo de forma dinámica a los cambios de la economía globalizada.

Por otra parte de acuerdo con Del Río, (2015) es importante la búsqueda de prácticas que permita evidenciar el camino que se debe seguir en una propuesta de negocio, lo que lleva a reconocer la importancia del modelo DIE como herramienta que pretende dar respuesta a las necesidades de la región.

Para Rodríguez et al. (2016) en Colombia el emprendimiento se debe apoyar en procesos de investigación desde la universidades jugando un papel calve en la formación de nuevos emprendedores que contribuyan a la gestión empresarial del país. Por tal razón para la UFPSO es de suma importancia fortalecer la aplicación del modelo DIE desde el programa de zootecnia desde el desarrollo de la línea administrativa de su malla curricular, ideando además procesos como electivas y opción para trabajo de grado la construcción de planes de negocio, además de participar en ferias empresariales dentro y fuera de la misma institución, buscando que sus estudiantes consoliden sus proyectos para la creación de nuevas empresas.

\section{LITERATURA CITADA}

ASCANIO, C. M., MONSAlVE, E. J. B., MUJICA, K. J. S., CARRASCAL, B. L. V., \& LEYVA, N. C. Evaluación financiera para la implementación de la semimecanización del sistema de transporte interno de la mina Shipio, 2017. Respuestas, 23(1), 119-126. 2018. \{En línea $\}.\{8$ de Abril de 2019$\}$ Disponible en: https://cutt.ly/6yJNDEw

BALANKO, G. Cómo preparar un plan de negocios exitoso. Editorial Mc Graw.2008

BARSKY, M. V. Plan de marketing para la sucursal Martinoli de la empresa Zárate (Doctoral dissertation). 2016. \{En línea $\}.\{18$ de Abril de 2019\} Disponible en: https://cutt.ly/5yJNVpl

BRAVO, B. Plan de negocios (Doctoral dissertation, Universidad Nacional de Cuyo. Facultad de Ciencias Económicas).2015. \{En línea $\}$. $\{18$ de Abril de 2019\} Disponible en: https://cutt.ly/0yJN6Ln

CAMPO, C. H. G., \& ALBARRACÍN, É. J. G. Modelo de Emprendimiento en Red-MER. Aplicación de las teorías del emprendimiento a las redes empresariales. Academia. Revista latinoamericana de administración, 
(40), Pp. 13-31.2008.\{En línea $\}.\{5$ de Abril de 2019\} Disponible en: $\underline{\text { https://cutt.ly/xyJNtwj }}$

CAMPOS RIAÑO, M., \& GÓMEZ CAMELO, Y. L. El emprendimiento en las mallas curriculares de los programas de administración de empresas de Bogotá Colombia. 2016.\{En línea $\}.\{29$ de Marzo de 2019\} Disponible en: https://cutt.ly/uyJNWfW

CAMPOS, H. M.¿Creadores de emprendedores o creadores de empleados?: El modelo educativo emprendedor del Tecnológico de Monterrey. In XI Congreso de Ingeniería de Organización (pp. 0601-0610). 2007. \{En línea\}.\{9 de Marzo de 2019\} Disponible en: https://cutt.ly/SyJB5Oa

CASTRO AMAYA, F. E. Guía técnica institucional para elaborar un plan de negocios.2017. \{En línea $\}$. $\{9$ de Marzo de 2019\} Disponible en: https://cutt.ly/ZyOP100

CEDEÑO VELASCO, A. P., CRISTOBAL, A., ROBERTO, L., \& VILLEGAS ALAVA, M. A. Las estrategias gerenciales como base fundamental para la administración en los negocios. Revista Universidad y Sociedad, 11(5), Pp. 191-200.2019. \{En línea\}. \{9 de Marzo de 2019\} Disponible en: https://cutt.ly/0yJMx85

CHACÓN, A. M. L., RODRÍGUEZ, G. A. A., VELANDIA, J. A. P., \& CARRASCAL, B. L. V. Análisis de aplicación móvil para la comercialización de productos publicitarios a través de plataformas IOS y Android. Revista CONVICCIONES, 5(9), Pp.58-61. 2018. \{En línea $\}.\{29$ de Marzo de 2019\} Disponible en: https://cutt.ly/lyJMgw0

CHAMORRO, E. T., CEBALlOS, H. O., \& VILLA, J. J. B. Política de emprendimiento en Colombia, 20022010. Estudios gerenciales, 29(128). 2013. Pp. 274-283. \{En línea $\}.\{14$ de Marzo de 2019\} Disponible en: https://cutt.ly/7yJBDkt

COLOMBIA. Congreso de la República. (ene., 2006) Ley 1014 de 2006: De fomento a la cultura del emprendimiento. Diario Oficial 46164. \{En línea $\}.\{24$ de Marzo de 2019\} Disponible en: https://cutt.ly/qyJBHdH

COLOMINA Climent, E., \& Yáñez Muñoz, L. Herramientas Para El Análisis Y Validación De Ideas De Negocio (Toolkit for Analysis and Validation of Business Ideas). Revista Global de Negocios, 2(4), $105-$ 123.2014. \{En línea\}. \{8 de Marzo de 2019\} Disponible en: https://cutt.ly/OyJNjJL

DEL RÍO, A. Z. El emprendimiento como elemento de formación universitaria: en busca de nuevas propuestas. Creatividad y sociedad: revista de la Asociación para la Creatividad, (23), Pp.182-199. 2015. \{En línea $\}$. 228 de Marzo de 2019\} Disponible en: https://cutt.ly/VyJMTcR

DUARTE, T., \& TIBANA, M. R. Emprendimiento, una opción para el desarrollo. Scientia et technica, 15(43), Pp. 326-331.2009

FERREIRA-HERRERA, D. C.El modelo Canvas en la formulación de proyectos. Cooperativismo \& Desarrollo, 23(107). 2015. \{En línea\}.\{13 de Marzo de 2019\} Disponible en: https://cutt.ly/kyJMGy3

FORERO, J. D. El sector del calzado en el barrio El Restrepo, Bogotá. Un análisis de caso a la luz de los sistemas productivos locales. Equidad y Desarrollo, 1(21), Pp. 97-123. 2014. \{En línea $\}$ \{19 de Marzo de 2019\} Disponible en: https://cutt.ly/syJNu1m

FUENTES PALOMINO, B., MARTINEZ DALlORSO, J. P., ÑAHUES CARDOSO, A. G., RAMOS 
LLIUYACC,A., \& ECHEVARRIA REYES, J. P.Plan de negocios:" Desarrollo de una aplicación que permite reunir dinero entre amigos". 2019. \{En línea\}. \{29 de Marzo de 2019\} Disponible en:https://cutt.ly/5yOPIE4

GALVIS, F. S. Fomento del emprendimiento a través de actividades académicas e investigativas. Tendencias, 18(2), Pp.183-196.2017.\{En línea\}.\{9 de Marzo de 2019\} Disponible en: https://cutt.ly/IyJNbRU

GÓMEZ, H. J., \& MITCHELL, D. Innovación y emprendimiento en Colombia-Balance, perspectivas y recomendaciones de política: 2014-2018. 2014.\{En línea $\}.\{4$ de Marzo de 2019\} Disponible en: https://cutt.ly/OyJBY08

GÓMEZ, M. J. A., SUÁREZ, C. H., \& CARRASCAL, B. L. V. Influencia de la adquisición de la marca Samsung celulares en la ciudad de Cúcuta. Revista CONVICCIONES, 5(9), Pp. 53-57.2018. \{En línea $\}$. 7 de Marzo de 2019\} Disponible en: https://cutt.ly/FyJM2lo

HERNÁNDEZ SÁNCHEZ, J., \& RODRÍGUEZ SOTO, J. R. Cuestionamientos sobre los esfuerzos en la implementación del emprendimiento en Colombia, una mirada desde el Estado, la academia y las agremiaciones.2013. \{En línea $\}.\{6$ de Marzo de 2019\} Disponible en: https://cutt.ly/syJBNKR

HOYOS-PATIÑO, J. F., BERMÚDEZ-GUTIÉRREZ, E., HERNÁNDEZ-VILLAMIZAR, D. A., \& VELÁSQUEZ-CARRASCAL, B. L. Aplicación del protocolo Welfare Quality ${ }^{\circledR}$ en criaderos equinos para determinar el grado de bienestar animal. Mundo FESC, 9(18), Pp. 24-30. 2019. \{En línea\}.\{5 de Abril de 2019 \} Disponible en: https://cutt.ly/gyJNs0U

KIRBERG, A. S. Emprendimiento exitoso. Ecoe Ediciones. 2014. \{En línea $\}.\{4$ de Abril de 2019\} Disponible en: https://cutt.ly/5yJBOgA

KIRKBERG, A. S. Creatividad, Innovación Y Marketing: Claves del Éxito Empreendedor. Caderno Profissional de Marketing-UNIMEP, 1(1), Pp. 64-74.2013. \{En línea\}.\{24 de Abril de 2019$\}$ Disponible en: https://cutt.ly/OyOPcWH

LÓPEZ LÓPEZ, F. Desarrollo del modelo de negocio Canvas en la tienda de productos alimenticios Víveres Ruiz de la parroquia Checa (Trabajo de titulación). Universidad Tecnológica Israel, Quito, Ecuador. 2017

LUNA, K. Y. R., OLIVAREZ, K. V. G., \& CARRASCAL, B. L. V. Moda artesanal sostenible elaborada por comunidades étnicas colombianas. Revista CONVICCIONES, 5(9), Pp. 13-21.2018. \{En línea $\}$. 17 de Abril de 2019 \} Disponible en: https://cutt.ly/1yJ1e4W

MACKINLAY, M., \& SABBAGH, A. Método de la innovación creativa, El: Un sistema para generar ideas y transformarlas en proyectos sustentables. Ediciones Granica. 2011. \{En línea\}.\{7 de Abril de 2019\} Disponible en: ttps://n9.cl/qhlg

MADARIAGA, H., \& ESTHER, J. EMPRENDIMIENTO E INNOVACIÓN: DISEÑA Y PLANEA TU NEGOCIO. 2019. \{En línea $\}.\{7$ de Abril de 2019\} Disponible en: https://cutt.ly/xyJNf3i

MERCEDES, P. A., GERMÁN, R. G., \& MARCELA, L. P. L. Emprendimiento y creatividad: Aspectos esenciales para crear empresa. Ecoe Ediciones.2017. \{En línea $\}.\{16$ de Abril de 2019\} Disponible en: https://cutt.ly/CyI477s

MINISTERIO DE COMERCIO, INDUSTRIA Y TURISMO.Manual para la elaboración de planes de 
negocios. Bogotá, Colombia: Ventures. 2010. \{En línea\}.\{7 de Abril de 2019\} Disponible en: https://cutt.ly/myJNTTO

MORA-VILLAMIZAR, D. A., MORALES-PÉREZ, K. T., BARRIENTOS-MONSALVE, E. J., \& VELÁZQUEZ-CARRASCAL, B. L. Análisis de la competitividad entre las empresas los olivos y la esperanza en Cúcuta, Norte de Santander-según las cinco fuerzas de Michael Porter. Revista CONVICCIONES, 6(11), Pp.69-75.2019. \{En línea $\}.\{6$ de Abril de 2019\} Disponible en: https://cutt.ly/WyJ1x7k

MORCELA, O. A., CABUT, M., \& PETRILLO, J. D. Participación del Observatorio Tecnológico OTEC en la formulación de proyectos PICT Start Up de la UNMdP. In XI Congreso de Ingeniería Industrial y I Congreso Internacional de Ingeniería-COINI 2018. \{En línea $\}.\{27$ de Abril de 2019\} Disponible en: https://cutt.ly/WyOPxfN

PATIÑO, J. F. H., \& GÓMEZ, R. A. CARACTERIZACIÓN DE LAS TENDENCIAS EN LA DOMA DE CABALLOS DE SILLA COLOMBIANO. FAGROPEC-Facultad de Ciencias Agropecuarias, 8(1). 2016. \{En línea\}.\{7 de Abril de 2019\} Disponible en: https://cutt.ly/yyJNpeH

PÉREZ, M., \& DARÍO, H.Las curvas en" S" como herramienta para evaluar el desempeño de las innovaciones en la formación del programa jóvenes rurales emprendedores-Sena. 2014. \{En línea $\}$. $\{20$ de Abril de 2019\} Disponible en: $\underline{\text { https://cutt.ly/TyJ1RNZ }}$

RENDÓN, Ó. H. P. Modelo del Plan de Negocios: Para Micro y Pequeña Empresa. Grupo Editorial Patria. 2014. \{En línea $\}$. $\{18$ de Abril de 2019\} Disponible en: https://cutt.ly/NyIlp2h

RODRÍGUEZ, J. G., RIAGA, M. C. O., GAITÁN, S. M. R., \& BOCANEGRA, P. M. S. Influencia de la universidad en la creación de empresa. Boletín Redipe, 5(2), Pp. 70-78.2016. \{En línea $\}.\{5$ de Abril de 2019$\}$ Disponible en: $\underline{\text { https://cutt.ly/4yJ1Cqp }}$

RODRÍGUEZ, L. Bogotá empresarial: desarrollo de la capacidad emprendedora y asociativa en los colegios oficiales de Bogotá. Universidad Autónoma de Colombia, Instituto Superior de Pedagogía. Bogotá, 2006. pág. 15.

RUBIO-CANO, B. S., MARTÍNEZ-ARBOLEDA, S. A., REYES-LINDARTE, J. A., IBARRA, D. A. P., VELÁSQUEZ-CARRASCAL, B. L., \& MARULANDA-ASCANIO, C. Planeación Para La Jubilación. Revista CONVICCIONES, 6(11), Pp. 76-84. 2019. \{En línea $\}.\{14$ de Abril de 2019$\}$ Disponible en: https://cutt.ly/XyJ0qqz

SANTANDREU MASCARELL, C., CANÓS DARÓS, L., \& MARÍN-ROIG RAMÓN, J. Business Model Canvas y redacción del plan de negocio.2014. \{En línea $\}.\{4$ de Abril de 2019\} Disponible en: https://cutt.ly/JyJ19d3

SCHNARCH, A. Emprendimiento exitoso: cómo mejorar su proceso y gestión. Ecoe Ediciones. 2014. \{En línea $\}.\{5$ de Marzo de 2019\} Disponible en: https://n9.cl/1g27

STUTELY, R. Plan de negocios: la estrategia inteligente. Pearson Educación. 2000.\{En línea $\}$. 17 de Marzo de 2019 \} Disponible en: https://cutt.ly/ayI3182

TRIMI, S., \& BERBEGAL-MIRABENT, J. Business model innovation in entrepreneurship. International Entrepreneurship and Management Journal, 8(4), Pp.449-465. 2012. \{En línea\}.\{15 de Marzo de 2019$\}$ 
Disponible en: $\underline{\text { https://cutt.ly/ryJNzPy }}$

URIBE, J. A. F. Plan de negocio: para pequeñas empresas. Ediciones de la U.2015. \{En línea $\}$. \{26 de Marzo de 2019\} Disponible en:https://cutt.ly/syI90xQ

VARGAS URZOLA, A. Modelo para la elaboración de un plan de negocios para las empresas pequeñas y medianas. Estudios Gerenciales, 18(82), Pp. 93-108. 2002. \{En línea\}.\{7 de Marzo de 2019\} Disponible en:https://cutt.ly/nyOPzhV

VÁSQUEZ, R. D. Antecedentes del emprendimiento-caso colombiano. Unaciencia, 4(7), 2011. Pp. 9-19.\{En línea $\}.\{5$ de Marzo de 2019$\}$ Disponible en: https://cutt.ly/LyJBLPE

VÁZQUEZ, J. M. S., ELORZA, M. L. V., \& PINZÓN, P. A. Balanced scorecard para emprendedores: desde el modelo canvas al cuadro de mando integral. Revista de la Facultad de Ciencias Económicas: Investigación y reflexión, 24(1), Pp. 37-47.2016. \{En línea\}.\{9 de Marzo de 2019\} Disponible en:https://cutt.ly/yyJ0Wuu

VILLARÁN, K. W. Plan de negocios. Herramientas para evaluar la viabilidad de un negocio, USAID Perú y Ministerio de la Producción, Perú.2009. \{En línea\}.\{10 de Marzo de 2019\} Disponible en: https://cutt.ly/gyJ0Qha 